\title{
ERRATUM
}

\section{Erratum to: Evaluation of the Efficacy of Sorafenib on Overall Survival in Patients with Hepatocellular Carcinoma using FT Rate: A Devised Index}

Takamasa Ohki (D) Mayuko Kondo · Yuki Karasawa · Satoshi Kawamura •

Shuuya Maeshima · Kentaro Kojima • Michiharu Seki •

Nobuo Toda $\cdot$ Yoshinobu Shioda $\cdot$ Kazumi Tagawa

Published online: May 26, 2017

(C) The Author(s) 2017. This article is an open access publication

Erratum to: Adv Ther

DOI 10.1007/s12325-017-0524-9

The authors of the above mentioned paper would like to make the following amendment to the author affiliations.

Kazumi Tagawa's affiliation currently reads "Department of Surgery, Shioda Hospital, Katsuura City, Japan". The affiliation should read
"Department of Gastroenterology, Mitsui Memorial Hospital, Tokyo, Japan".

Finally, the authors would like to make the following amendments to Fig. 2. The x-axis labels for both Fig. 2a and Fig. 2b currently read "Time from TACE refractory (years)". The labels should read "Time (years)". The corrected Fig. 2 is provided here.

The online version of the original article can be found under doi:10.1007/s12325-017-0524-9.

T. Ohki (₫) · M. Kondo · Y. Karasawa ·

S. Kawamura - S. Maeshima - K. Kojima · M. Seki ·

N. Toda $\cdot$ K. Tagawa

Department of Gastroenterology, Mitsui 20

Memorial Hospital, Tokyo, Japan

e-mail: lily.rover220@gmail.com

Y. Shioda

Department of Surgery, Shioda Hospital, Katsuura

City, Japan 
(a)

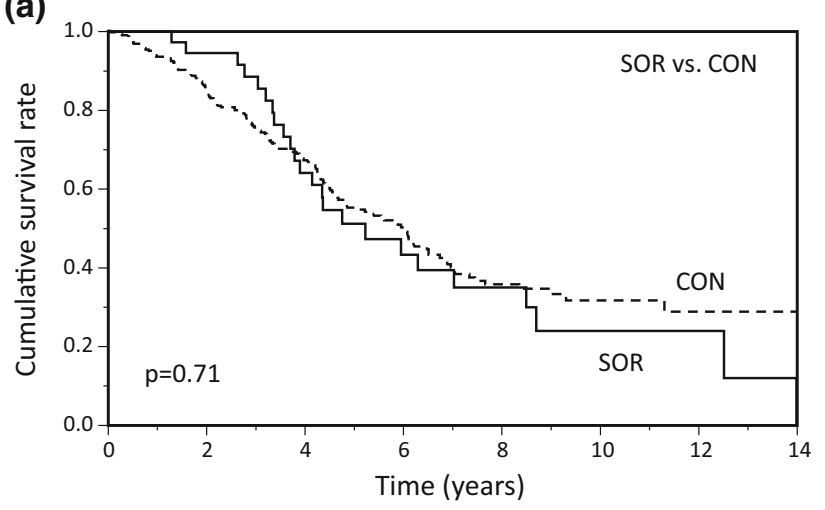

(b)

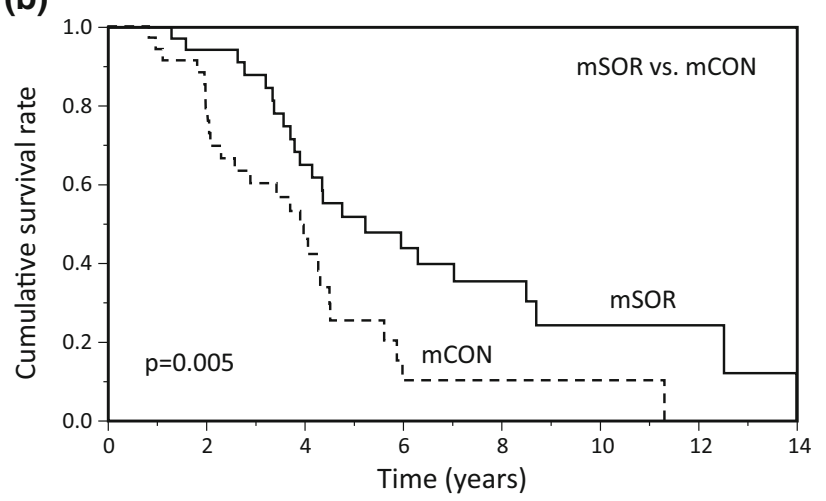

Fig. 2 Cumulative survival rates for a SOR vs. CON and b mSOR vs. mCON. CON transcatheter arterial chemoembolization, $m C O N$ matched transcatheter arterial chemoembolization, $m S O R$ matched sorafenib, SOR sorafenib

Open Access. This article is distributed under the terms of the Creative Commons Attribution-NonCommercial 4.0 International License (http://creativecommons.org/licenses/by-nc/4. $0 /)$, which permits any noncommercial use, distribution, and reproduction in any medium, provided you give appropriate credit to the original author(s) and the source, provide a link to the Creative Commons license, and indicate if changes were made. 